\title{
A Bio-inspired Aggregation with Robot Swarm using Real and Simulated Mobile Robots
}

DOI:

10.1007/978-3-319-96728-8_27

\section{Document Version}

Accepted author manuscript

Link to publication record in Manchester Research Explorer

\section{Citation for published version (APA):}

Ramroop, S., Arvin, F., Watson, S., Carrasco, J., \& Lennox, B. (2018). A Bio-inspired Aggregation with Robot Swarm using Real and Simulated Mobile Robots. In M. E. Giannaccini, M. Giuliani, \& T. Assaf (Eds.), Towards Autonomous Robotic Systems - 19th Annual Conference, TAROS 2018, Proceedings (pp. 317-329). (Lecture Notes in Computer Science (including subseries Lecture Notes in Artificial Intelligence and Lecture Notes in Bioinformatics); Vol. 10965 LNAI). Springer Nature. https://doi.org/10.1007/978-3-319-96728-8_27

\section{Published in:}

Towards Autonomous Robotic Systems - 19th Annual Conference, TAROS 2018, Proceedings

\section{Citing this paper}

Please note that where the full-text provided on Manchester Research Explorer is the Author Accepted Manuscript or Proof version this may differ from the final Published version. If citing, it is advised that you check and use the publisher's definitive version.

\section{General rights}

Copyright and moral rights for the publications made accessible in the Research Explorer are retained by the authors and/or other copyright owners and it is a condition of accessing publications that users recognise and abide by the legal requirements associated with these rights.

\section{Takedown policy}

If you believe that this document breaches copyright please refer to the University of Manchester's Takedown Procedures [http://man.ac.uk/04Y6Bo] or contact uml.scholarlycommunications@manchester.ac.uk providing relevant details, so we can investigate your claim.

\section{OPEN ACCESS}




\title{
A Bio-inspired Aggregation with Robot Swarm using Real and Simulated Mobile Robots
}

\author{
Sarika Ramroop, Farshad Arvin, Simon Watson, Joaquin Carrasco-Gomez, and \\ Barry Lennox \\ Robotics for Extreme Environments Lab (REEL) \\ School of Electrical \& Electronic Engineering \\ The University of Manchester, Manchester, M13 9PL, UK \\ sarika.ramroop@postgrad.manchester.ac.uk
}

\begin{abstract}
This paper presents an implementation of a bio-inspired aggregation scenario using swarm robots. The aggregation scenario took inspiration from honeybee's thermotactic behaviour in finding an optimal zone in their comb. To realisation of the aggregation scenario, real and simulated robots with different population sizes were used. Mona, which is an open-source and open-hardware platform was deployed to play the honeybee's role in this scenario. A model of Mona was also generated in Stage for simulation of aggregation scenario with large number of robots. The results of aggregation with real- and simulated-robots showed reliable aggregations and a population dependent swarm performance. Moreover, the results demonstrated a direct correlation between the results observed from the real robot and simulation experiments.
\end{abstract}

Keywords: Aggregation, Swarm Robotics, Bio-inspired, Open-source.

\section{Introduction}

Swarm robotics [1] allows many simple robots to work together to carry out tasks that they would not be able to do individually. Many swarm algorithms are based on biological systems such as swarms of bees [2], flocks of birds [3], or ants colonies [4]. Using swarms can allow for greater efficiency as sub-tasks can be carried out in parallel by different groups of robots [5]. Swarms are also generally robust as the failure of a few robots in the collective has little effect of achieving the overall objective. Swarms require large numbers of robots, which means that experimentally testing algorithms can be difficult due to the cost and time required to set up and carry out the experiments. As such, before deploying algorithms on the actual robots, it is beneficial to carry out simulations to estimate an algorithm's performance.

Mona [6] is an open-hardware and open-source robot developed at the University of Manchester for swarm robotic research and teaching. In keeping with the design philosophy of swarm robots, its base model is relatively simple; its only built-in sensors are infrared (IR) sensors at the front of the robot and 
encoders for the wheels. Additional sensors, such as vision system [7], light sensors [8], inductive charging [9], or radio frequency (RF) modules can be added as there are available input/output ports. Mona was developed in 2017 and prior to this work, there was no simulation environment for it. Any algorithms that needed to be testing required direct deployment on the hardware. Mona is also used for robot education hence a simulation environment would enable use of the robot when physical devices are not available.

The aim of this work was to select a suitable simulation environment for Mona, modelling the robot's physical and control structure, implementing aggregation algorithm for testing, and conducting comparisons of the simulated behaviour with the actual behaviour when implemented in hardware.

\section{Background and Related Work}

This section presents the background and related work for the proposed aggregation scenario and corresponding swarm robot platforms and their simulation environments.

\subsection{The Mona Swarm Robot Platform}

Mona [6] is an open-source low-cost miniature mobile robot which has been developed at the University of Manchester for swarm robotic research and teaching. It's primary advantage compared to other existing platforms is its inbuilt inductive charging system which allows for perpetual swarm experiments to be run [10].

Fig 1 (left) shows the overhead view of the Mona robot. It is a differential drive robot [11] with a maximum velocity of approximately $7 \mathrm{~cm} / \mathrm{s}$ and makes use of an ATmega 328 chip. This can be programmed using the Arduino development environment after uploading a boot-loader to the microcontroler. There are three IR sensors which allow to robot to carry out obstacle detection [12]. After testing, the maximum range was determined to be $45 \mathrm{~mm}$, with an angular range of up to $80^{\circ}$. The IR sensors also enable robot identification. Additionally, a light dependent resistor (LDR) shown in Fig. 1 (right) allows for light intensity measurement.

\section{$2.2 \quad$ Aggregation Algorithm}

BEECLUST (Fig. 2) is a bio-inspired swarm aggregation algorithm which was based by the tendency of young honeybees to gather in areas with temperatures between $34^{\circ} \mathrm{C}$ and $38^{\circ} \mathrm{C}$ [2]. Robot implementations of BEECLUST have used light intensity $[2,9,13]$ or sound signals $[14,15]$ instead of temperature, with the robots clustering in areas (cues) with the highest intensity of either source.

Based on the aggregation scenario, the robot is in one of four states:

1. forward: robot moves forward in a straight line, while checking for obstacles 

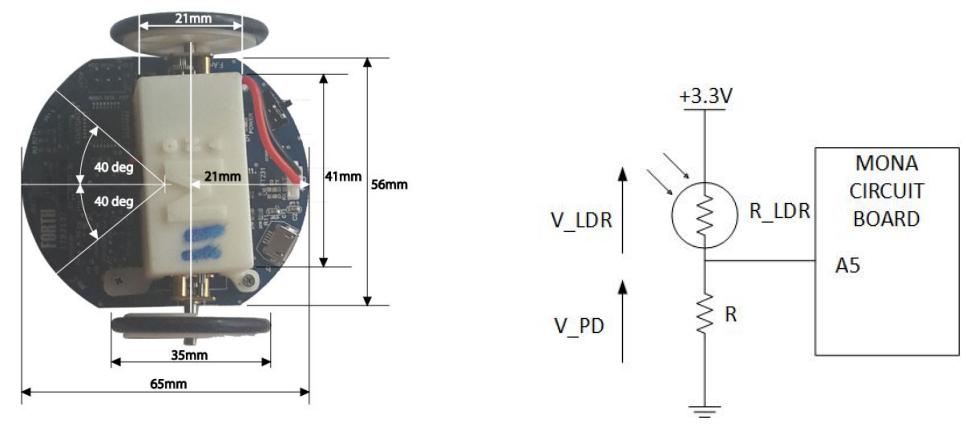

Fig. 1: (left) An overhead view of the Mona robot (right) light sensing circuit

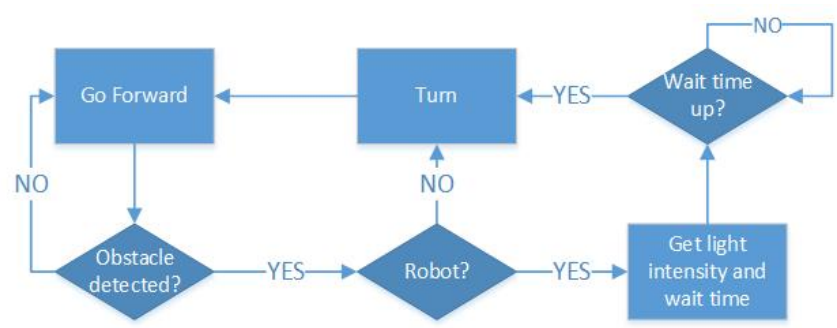

Fig. 2: BEECLUST algorithm state diagram for a single robot

2. obstacle: checks if an obstacle is a robot or wall

3. wait: if the obstacle is another robot, the robot waits for length of time based on the measured light intensity

4. turn: the robot turns to avoid an obstacle/robot and then returns to forward.

BEECLUST has previously been implemented by platforms such as Jasmine [2] and Colias [9] which have similar characteristics as shown in Table 1.

Table 1: Comparison of different open-source swarm robotic platforms

\begin{tabular}{lcccc}
\hline Robot & $\begin{array}{c}\text { Size }(\text { diam. or } \\
l \times w)(\mathrm{mm})\end{array}$ & $\begin{array}{c}\text { Type of } \\
\text { motion }\end{array}$ & Sensors & Sensor range \\
\hline Mona [6] & 65 & Diff. drive & $3 \mathrm{IR}$ & $45 \mathrm{~mm}, 180^{\circ}$ \\
Jasmine [2] & $23 \times 23$ & Diff. drive & $6 \mathrm{IR}$, light & $60 \mathrm{~mm}, 360^{\circ}$ \\
Colias [16] & 40 & Diff. drive & $6 \mathrm{IR}$, light & $150 \mathrm{~mm} 360^{\circ}$ \\
\hline
\end{tabular}

\subsection{Simulators for Swarm Robotics}

The most popular simulators for swarm robotics include Gazebo, ARGoS, Player/Stage and Webots $[17,18]$. This section provides a review of these environments with respect to their use for simulations of BEECLUST with Mona. 
Gazebo [19] is widely used for simulating robot behaviour; swarm or otherwise [18]. It provides a 3D visualisation environment and custom robots can be created in it. Gazebo is open-source and code written for robot applications can often be ported easily from Gazebo to the actual robot. Due to its popularity [17], a lot of support can be found from the active online community. However, because Gazebo renders its simulations in a 3D environment, it can be relatively computationally heavy. For swarm applications with many robots (greater than 10), the performance could be too slow.

ARGoS [20] is widely used simulator which also provides 3D simulations. It was developed as part of the Swarmanoid project [21] and is therefore used in several different swarm related research such as $[22,23]$. As a result, ARGoS tends to be used mainly in relation to the foot-, hand- and eye-bot robots. While ARGoS allows for fast performance with many robots, it is difficult to customise for a user-defined robot.

Webots [24] and Stage [25] are among the most recognised simulation platforms as both have existed for several years. Webots presents the same problem as Gazebo; that is, it is not suitable for large numbers of robots.

Stage [25] is described as a $2.5 \mathrm{D}$ simulator and can simulate robots through a range of customisable sensor modules and actuators. Environments can be simulated to include different colour cues and fixed and movable obstacles. In general, one of the disadvantages of Stage is that it only allows for differential drive robot.

Table 2 shows a comparison of the simulation environments. Due to the relative ease of simulating robots in Stage and creating the environments, and confirmation that BEECLUST can be simulated, Stage was selected to build the simulations for the Mona robots.

\section{Experimental Procedure}

\subsection{Arena Setup}

All hardware tests utilised a $72 \mathrm{~cm} \times 44.8 \mathrm{~cm}$ arena with $3.2 \mathrm{~cm}$ walls (Fig. 3). LED bulbs were used to create the cue since they emit less IR radiation that halogen bulbs. This eliminates a potential source of IR interference to the robots' sensors.

Table 2: Comparison of different open-source swarm robotic platforms

\begin{tabular}{lccc}
\hline Simulator & Visualisation & Customisation & $\begin{array}{c}\text { Number of } \\
\text { Robots }\end{array}$ \\
\hline Gazebo [19] & $3 \mathrm{D}$ & Easy & Low \\
ARGoS [20] & $3 \mathrm{D}$ & Hard & High \\
Webots [24] & $3 \mathrm{D}$ & Easy & Low \\
Stage [25] & $2.5 \mathrm{D}$ & Easy & Very High \\
\hline
\end{tabular}



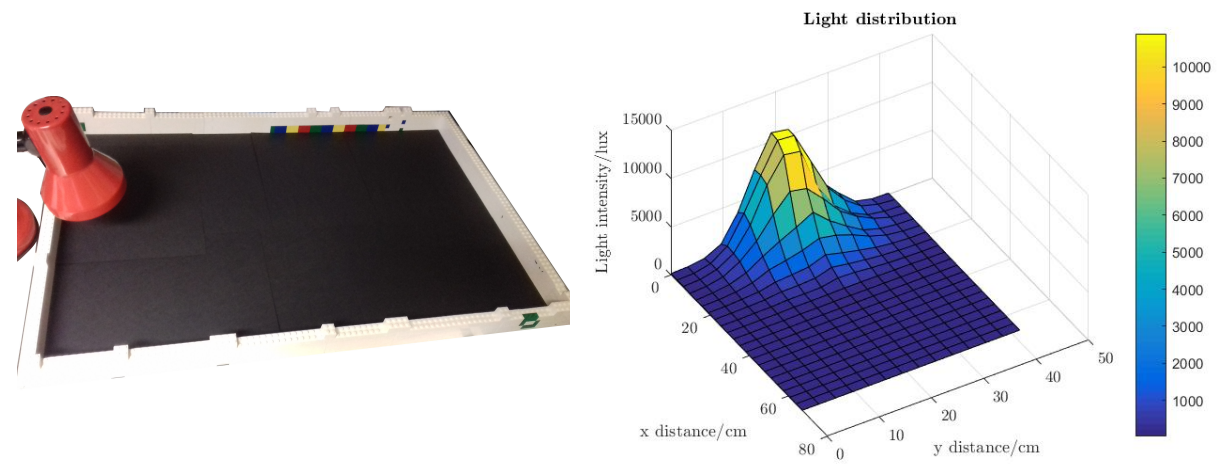

Fig. 3: (left) Lego arena with one light source and (right) 3D graph showing light intensity of the arena with one light source.

The arena walls were constructed with white Lego blocks as this surface reflects IR signals well so would be easily detected by the sensors. The arena's floor was covered in black cardboard. This absorbs IR signals and prevent IR and light from being reflected and affecting sensor readings.

The cue size was determined by Eq. 1, as adapted from [9]:

$$
a_{c}=\beta n a_{r}
$$

where $a_{c}$ is the area of the cue, $n$ is the number of robots, $a_{r}$ is the area occupied by a robot and $\beta$ is the ratio of cue to area occupied by the swarm.

Using the minimum $\beta$ value of two and the maximum number of robots of eight, a cue with radius of $16 \mathrm{~cm}$ was selected. This was also modelled in simulation. For a simulation only arena, a maximum of 50 robots was used. This required a cue radius of $46 \mathrm{~cm}$. The arena size with selected as $100 \mathrm{~cm} \times 160 \mathrm{~cm}$ to allow for this.

\subsection{Experiment 1: Static environment with single light source}

This experiment used a single light source at one end of the arena (Fig. 3). All the robots were turned on while the light was switched off and placed outside the general cue area. The light was then turned on. This was to allow all the robots to respond to the light at the same time.

The time taken for the robots to aggregate was recorded $\left(t_{a}\right)$. A robot was considered to be aggregated if it was in the cue while in the waiting state or enclosed by other robots in the waiting state. Aggregation was considered to be completed when $70 \%$ of the total number of robots were aggregated [9]. This experiment was carried out with increasing the number of robots to observe the effect. For the real-robot implementation $n \in\{3,4,5,6,7,8\}$ robots were used. This same number of robots was also used in simulation, but further tests were carried out with up to 50 robots. 
Table 3: Experimental values or range for variables and constants

\begin{tabular}{lll}
\hline Values & Description & Range/Values \\
\hline$n$ & Total number of robots & Real Robot: $\{3,4,5,6,7,8\}$ \\
& & Simulation: $\{10,20,30,40,50\}$ \\
$n_{a}$ & Number of aggregated robots & 0 to 50 \\
$s$ & Sensor value & 0 to 255 \\
$w$ & Wait time & 0 to $85 \mathrm{sec}$ \\
cruiseSpd & Forward speed & $4 \mathrm{~cm} / \mathrm{s}$ \\
safeDist & Minimum safe distance & $1 \mathrm{~cm}$ \\
turnSpd & Turn speed & $1 \mathrm{rad} / \mathrm{sec}$ \\
$r_{c}$ & Cue radius & $\{16,46\} \mathrm{cm}$ \\
$t_{a}$ & Aggregation time & 0 to $500 \mathrm{sec}$ \\
\hline
\end{tabular}

\subsection{Experiment 2: Static environment with two light sources}

This experiment is simular to Experiment 1, except two light sources with different light intensities were used. The light sources produced different cue sizes and were situated at opposite ends of the arena. The robots were placed outside the cue and the aggregation times were recorded. Aggregation was considered to be completed when $70 \%$ of the total number of robots were waiting in either one of the cues. This was also carried out with increasing numbers of robots.

Table 3 gives the definitions and values of any constants and variables used in the both Experiments 1 and 2. Each set of experiment was repeated 10 times and results were statistically analysed.

\section{Simulated Robot in Stage}

\subsection{Environment Model}

As shown in Fig. 3, the light source has maximum intensity at the centre. The cue was modelled in Stage using a colour gradient with shades of gray, from white (maximum intensity) to black (darkness). Each colour has an associated RGB value from which the $R$ value was scaled to a value between 0 and 255 to represent the light sensor measurement.

The arena walls were created from a '.png' image with a black square that was scaled to the desired length, width and height values. This was set to be recognized as an obstacle.

\subsection{Robot Model}

The physical model was created using the measurement from Fig. 1. The following details how the sensors and actuators were implemented.

1. Differential drive - built into the Stage position model

2. IR sensors for obstacle detection - the ranger module is used to implement a sensor with range of $45 \mathrm{~mm}$ and $80^{\circ}$. 
3. Light sensors - implemented using the blobfinder module, which returns colour values

4. IR sensors for robot detection - implemented using the fiducial model which is set to recognise objects assigned a value of " 1 ".

Fig. 4 shows the robot model in Stage, where the image on the left shows the different sensors and the image on the right shows how the fiducial model works to identify another robot.
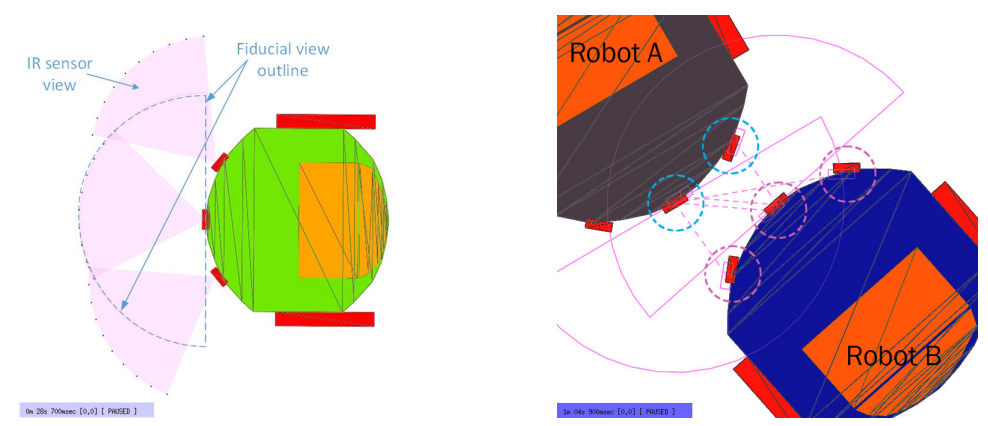

Fig. 4: (Left) robot sensor views and (right) The fiducial module on Robot A detects the sensors that are circled on Robot $B$ and vice versa).

\section{Results of Experiments}

\subsection{Experiment 1: Static environment with single light source}

Fig. 5 shows a randomly selected final aggregated positions of the Mona robots for real and simulated experiments where the total number of aggregated robots, $n_{a}$, is five.
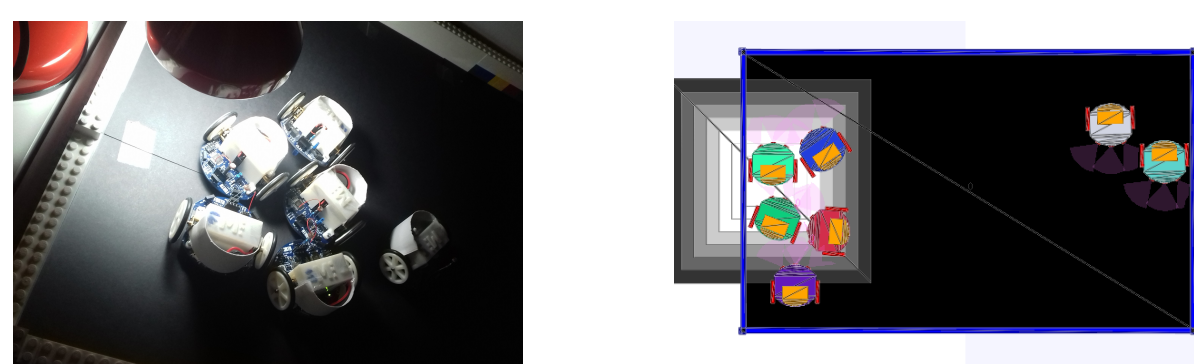

Fig. 5: Aggregation of 5 real robots $(n=7)$ with: (left) real-robots and (right) simulated robots. 
Table 4: Two-way ANOVA analysis for Experiment 1

\begin{tabular}{lcc}
\hline Source & $F$-value & $p$ value \\
\hline Number of Robots & 5.47 & 0.0005 \\
Experiment Type & 0.0048 & 0.945 \\
Robots * Experiment & 0.49 & 0.785 \\
\hline
\end{tabular}

Statistical analysis was carried out on the aggregation times obtained for both the real and simulated scenarios. The analysis of variance (ANOVA) and $F$-test method was used to disprove the null hypotheses, which states that the means for different conditions are the same. A high $F$-value (greater than 1 ) and a low $p$-value (less than 0.05 ) indicates that a factor significantly affects results. It does not indicate how it affects the result.

Two-way ANOVA was carried out where the factors were the robot population size $(n \in\{3,4,5,6,7,8\})$ and the type of experiment (real or simulated robots). Table 4 shows the $F$ - and $p$-values that were obtained.

The $F$-value for experiment type is less than 1 with a high $p$-value. This suggests that the null hypothesis cannot be disproven and that data from the real and simulated experiments are very similar. However, in general, this can also indicate an error with the data.

To account for this, one-way ANOVA was carried out with the factor as the experiment type, for each value of $n$. The $F$-values were between 0.01 and 0.80 while $p$-values were between 0.3971 and 0.9387 , indicating inability to disprove the null hypothesis. This validates the results of the two-way ANOVA analysis about the similarity of the real and simulated data. Hence, even though the simulation is not a perfect match, the results can be considered good enough to estimate the behaviour of the real system.

The second factor in Table 4 is the number of robots. The large $F$-value and small $p$-value indicate that population size significantly affects aggregation times. Fig. 6 shows the box and whisker plot of the times for the different population sizes. The results shows clearly that increasing the population size decreases the aggregation time. This occurs because BEECLUST depends on collisions in light for aggregation to take place. Most robots in the same arena increases the likelihood of collisions. This matches the observations by Schmickl et al. [2] and Arvin et al. [9] who both noted this improved performance with more robots.

Fig. 6 also shows that as the number of robots increases, the difference in aggregation time becomes smaller. This occurs becasue there is also an increased number of collisions outside of the light cue so robots need to reorient themselves continuously. The general observation was noted in [9], while Hereford [26] also observed this nonlinearity in the relationship between population size and aggregation time.

Fig. 7 shows the number of robots aggregated over time for $n=4$ and $n=8$. Only these two cases are considered as they should provide enough contrast to see the effects of increasing population size. 


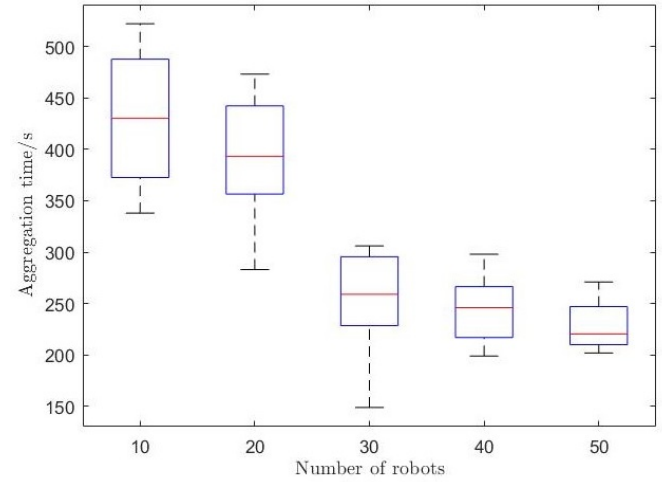

Fig. 6: Aggregation times for the simulated Experiment 1, using the larger arena
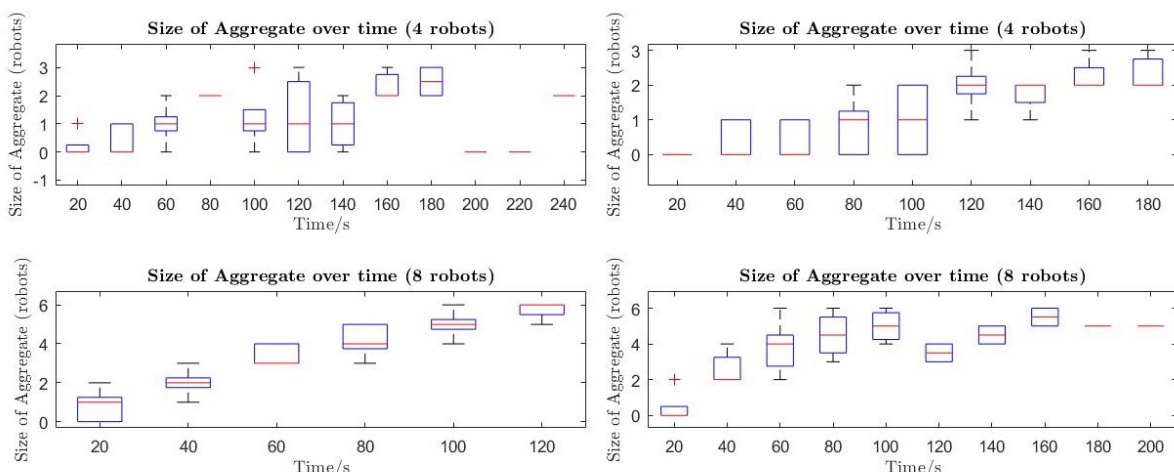

Fig. 7: Number of robots in the aggregate over time, for both (left) real robots and (right) simulated robots.

For case $n=4$, there is fluctuation in the number of robots over time as the enter and leave the aggregate. This is becasue there is a lower collision rate, so robots leave the waiting state before other robots are able to collide. In contrast, for $n=8$, there is a general increase in the aggregate size over time. This shows that a larger aggregate is more likely to increase in size since unaggregated robots have a higher probability of colliding with a robot in the wait state. Fig. 7 (right) shows that when robots leave the aggregate, the overal aggregation time increases. This behaviour matches the results obtained by Arvin et al [9]. Their aggregation graphs for dynamic environments show display similar results. Note only the initial aggregation can be compared here.

\subsection{Experiment 2: Static environment with two light sources}

The box and whisker diagram in Fig. 8 shows the collective decision of the swarm for the larger arena. The smaller population sizes were not considered 
here since the consensus is less obvious due to fewer collisions being required for aggregation.

Fig. 8: Robots per cue, for cases $n \in\{10,30,50\}$

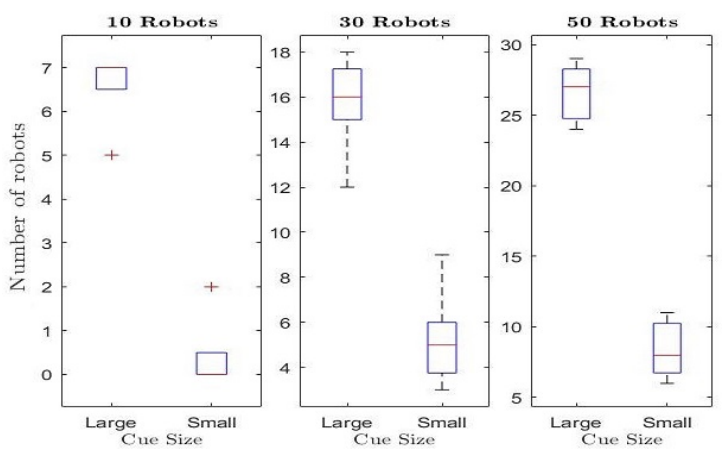

In every case, the majority of robots aggrgate around the larger cue. This behaviour is observed in [2] and [9]. The results from Experiment 1 show enough similarity between real and simulated performances to conclude that if this was implemented in hardware, there would also be a preference to aggregate around the global optimum. Therefore, this BEECLUST property is also replicated by the Mona robots.

Table 5 shows the results of two-way ANOVA analysis where the factors were number of robots and number of cues. The data used is for $n \in\{3,4,5,6,7,8\}$.

Table 5: Two-way ANOVA analysis for Experiment 2

\begin{tabular}{lcc}
\hline Source & $F$-value & $p$ value \\
\hline Number of Robots & 8.52 & $<0.00001$ \\
Number of Cues & 0.3776 & 0.5418 \\
Robots * Cues & 0.1740 & 0.9710 \\
\hline
\end{tabular}

The $F$ - and $p$-values show that the number of robots has a significant effect on aggregation. This was also observed with Experiment 1, so is expected.

The small $F$-value and large $p$-value for number of cues means the null hypothesis cannot be discredited. This differs from the observations in [9]. They find that cue size does affect aggregation time, though to a less degree than population size. Two cues behave as a larger single cue.

It is possible that the population sizes in this analysis are too small to observe this difference and there is not a large enough difference between the population sizes. As a result, a box and whisker diagrams were also done for $n \in\{10,30,50\}$ with single and double cues.

This more closely matches the observations in other works as the aggregation time with two cues is generally less than with a single cue. 

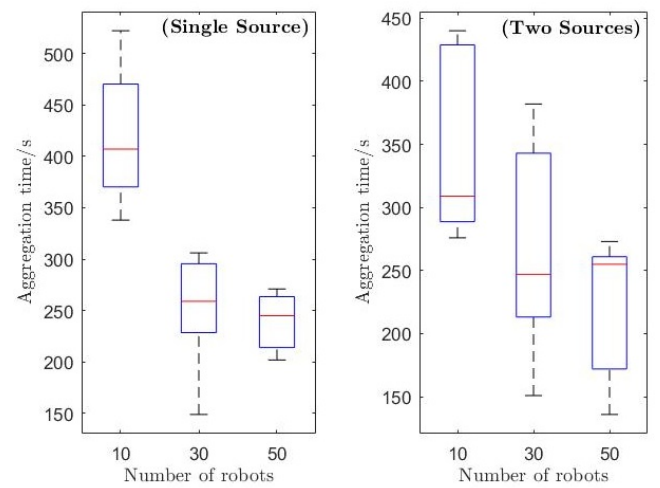

Fig. 9: Aggregation times for simulated Experiment 1 and simulated Experiment 2 for larger $n$ values

\section{Conclusion}

In this work, the LDR potential divider circuit was added to the Mona robots to allow them to execute the BEECLUST algorithm. Even though the short IR sensor range affects the performance, the robots were still able to achieve aggregation under a light source. The observed behaviour was comparable with other work that have been previously carried out using this algorithm. Using the ANOVA statistical method, it was determined that the Stage simulations provide behaviour close enough to the real robots' performance. For the future work, dynamic environments using single and multiple cues will be investigated.

\section{Acknowledgement}

This work was supported by the EPSRC (Project No. EP/P01366X/1).

\section{References}

1. Hamann, H.: Swarm Robotics: A Formal Approach. Springer (2018)

2. Schmickl, T., Thenius, R., Moeslinger, C., et al.: Get in touch: cooperative decision making based on robot-to-robot collisions. Autonomous Agents and Multi-Agent Systems 18(1) (2009) 133-155

3. Turgut, A.E., Çelikkanat, H., Gökçe, F., Şahin, E.: Self-organized flocking in mobile robot swarms. Swarm Intelligence 2(2) (2008) 97-120

4. Dorigo, M., Birattari, M., Stutzle, T.: Ant colony optimization. IEEE Computational Intelligence Magazine 1(4) (2006) 28-39

5. Şahin, E.: Swarm robotics: From sources of inspiration to domains of application. In: International workshop on swarm robotics. (2005) 10-20

6. Arvin, F., Espinosa, J., Bird, B., West, A., Watson, S., Lennox, B.: Mona: an affordable open-source mobile robot for education and research. Journal of Intelligent \& Robotic Systems (2018)

7. Hu, C., Arvin, F., Xiong, C., Yue, S.: Bio-Inspired Embedded Vision System for Autonomous Micro-Robots: The LGMD Case. IEEE Transactions on Cognitive and Developmental Systems 9(3) (2017) 241-254 
8. Arvin, F., Krajník, T., Turgut, A.E., Yue, S.: COS $\Phi$ : artificial pheromone system for robotic swarms research. In: IEEE/RSJ International Conference on Intelligent Robots and Systems (IROS). (2015) 407-412

9. Arvin, F., Turgut, A.E., Krajnk, T., Yue, S.: Investigation of cue-based aggregation in static and dynamic environments with a mobile robot swarm. Adaptive Behavior 24(2) (2016) 102-118

10. Arvin, F., Watson, S., Turgut, A., Espinosa, J., Krajník, T., Lennox, B.: Perpetual robot swarm: Long-term autonomy of mobile robots using on-the-fly inductive charging. Journal of Intelligent \& Robotic Systems (2017) 1-18

11. Arvin, F., Bekravi, M.: Encoderless position estimation and error correction techniques for miniature mobile robots. Turkish Journal of Electrical Engineering \& Computer Sciences 21(6) (2013) 1631-1645

12. Arvin, F., Samsudin, K., Ramli, A.: Development of IR-Based Short-Range Communication Techniques for Swarm Robot Applications. Advances in Electrical and Computer Engineering 10(4) (2010) 61-68

13. Arvin, F., Samsudin, K., Ramli, A.R., Bekravi, M.: Imitation of honeybee aggregation with collective behavior of swarm robots. International Journal of Computational Intelligence Systems 4(4) (2011) 739-748

14. Arvin, F., Turgut, A.E., Bazyari, F., Arikan, K.B., Bellotto, N., Yue, S.: Cue-based aggregation with a mobile robot swarm: a novel fuzzy-based method. Adaptive Behavior 22(3) (2014) 189-206

15. Arvin, F., Turgut, A.E., Bellotto, N., Yue, S.: Comparison of different cue-based swarm aggregation strategies. In: International Conference in Swarm Intelligence. (2014) 1-8

16. Arvin, F., Murray, J., Zhang, C., Yue, S.: Colias: An autonomous micro robot for swarm robotic applications. International Journal of Advanced Robotic Systems 11(7) (2014) 113

17. Ivaldi, S., Padois, V., Nori, F.: Tools for dynamics simulation of robots: a survey based on user feedback. arXiv:1402.7050 (2014)

18. Tan, Y., Zheng, Z.y.: Research advance in swarm robotics. Defence Technology 9(1) (2013) 18-39

19. Koenig, N., Howard, A.: Design and use paradigms for gazebo, an open-source multi-robot simulator. In: IEEE/RSJ International Conference on Intelligent Robots and Systems (IROS). Volume 3. (2004) 2149-2154

20. C. Pinciroli, et al.: ARGoS: a modular, parallel, multi-engine simulator for multirobot systems. Swarm Intelligence 6 (2012) 271-295

21. M. Dorigo, et al.: Swarmanoid: A novel concept for the study of heterogeneous robotic swarms. IEEE Robotics Automation Magazine 20(4) (2013) 60-71

22. M.A. Montes de Oca, et al.: Majority-rule opinion dynamics with differential latency: a mechanism for self-organized collective decision-making. Swarm Intelligence 5(3) (2011) 305-327

23. Ducatelle, F., Di Caro, G.A., Pinciroli, C., Gambardella, L.M.: Self-organized cooperation between robotic swarms. Swarm Intelligence 5(2) (2011) 73

24. Michel, O.: Cyberbotics ltd. webots: Professional mobile robot simulation. International Journal of Advanced Robotic Systems 1(1) (2004) 5

25. Vaughan, R.: Massively multiple robot simulations in stage. Swarm Intelligence 2(1) (2008) 189-208

26. Hereford, J.: Analysis of BEECLUST swarm algorithm. In: IEEE Symposium on Swarm Intelligence. (2011) 1-7 